Original

\title{
Amelioration of Nedaplatin-Induced Nephrotoxicity by Continuous Infusion in Rats
}

\author{
Takeki Uehara ${ }^{1}$, Noriko Tsuchiya ${ }^{1}$, Mikinori Torii ${ }^{1}$, \\ Jyoji Yamate $^{2}$, and Toshiyuki Maruyama ${ }^{1}$ \\ ${ }_{1}^{1}$ Developmental Research Laboratories, Shionogi \& Co., Ltd., 3-1-1 Futaba-cho, Toyonaka, Osaka 561-0825, Japan \\ 2 Department of Veterinary Pathology, Graduate School of Agriculture and Biological Science, Osaka Prefecture \\ University, 1-1 Gakuen-cho, Sakai, Osaka 599-8531, Japan
}

\begin{abstract}
The current experiment was conducted to evaluate whether prolongation of infusion time is useful for the amelioration of nedaplatin (NDP)-induced nephrotoxicity. Eight-week-old male rats were treated with $12 \mathrm{mg} / \mathrm{kg}$ NDP with the following dosing protocols, bolus injection, 1- or 4-hour continuous infusions (1hIF or 4hIF, respectively), and sacrificed 3 days after dosing. Urinary parameters were monitored on the day of the sacrifice, and the kidneys and femurs were removed for histopathological examination and bone marrow analysis. In the Bolus-NDP group, urine $\mathrm{pH}$ and specific gravity were decreased compared with the corresponding control group and glucosuria and occult blood were detected, while no abnormalities were noted in these urine parameters in the 4hIF-NDP group. Histopathological examination revealed slight to moderate renal lesions, such as single cell and/or focal necrosis in the epithelium of cortical tubules and collecting ducts in the renal papilla in the Bolus-NDP group. In the 1hIF- and 4hIF-NDP groups, there were clear reductions in the severity of renal tubular damage compared with the Bolus-NDP group, and the severity of renal tubular damage tended to reduce with increased infusion time. A TUNEL assay revealed that the numbers of TUNEL-positive cells in the 1hIF- and 4hIF-NDP groups were significantly lower than that in the Bolus-NDP group. There was a significant decrease in the number of TUNEL-positive cells in the 4hIF-NDP group compared with that in the 1hIF-NDP group. However, prolongation of the infusion time had no clear effect on the myelotoxicity of NDP. These results provide new evidence that prolongation of the infusion time is effective at minimizing the nephrotoxicity of NDP. (J Toxicol Pathol 2007; 20: 141-147)
\end{abstract}

Key words: amelioration, continuous infusion, nedaplatin, nephrotoxicity, rat

\section{Introduction}

Nedaplatin [cis-diammineglycolatoplatinum (II), NDP] is a second-generation antineoplastic platinum complex with reduced nephrotoxicity. It is now marketed in Japan as a drug, which may serve as a substitute for cisplatin [cisdiamminedichloroplatinum (II), CDDP] or even surpass it for use in combination with other drugs ${ }^{1-6}$. While nephrotoxicity occurs less frequently, the NDP dose is limited by the fact that it can cause myelosuppression, resulting in thrombocytopenia ${ }^{7}$.

Previously, we have reported that while NDP is clearly less nephrotoxic than CDDP in rats, it also induces nephrotoxicity, which is morphologically characterized by lysosomal hyperplasia, necrosis and hyperplasia of the

Received: 12 March 2007, Accepted: 1 June 2007

Mailing address: Takeki Uehara, Developmental Research

Laboratories, Shionogi \& Co., Ltd., 3-1-1 Futaba-cho, Toyonaka,

Osaka 561-0825, Japan

TEL: 81-6-6331-8241 FAX: 81-6-6332-6385

E-mail: takeki.uehara@shionogi.co.jp collecting duct epithelium and epithelium covering the renal papilla, with slight cortical lesions, such as proximal tubular dilatation and regeneration ${ }^{8}$. Therefore, attention must be paid to the nephrotoxicity of NDP during use, especially in patients with reduced renal function, even though it is a promising drug with reduced nephrotoxicity. The toxicological information currently available is limited and additional experimental studies focused on the amelioration of NDP's nephrotoxicity are necessary. Thus, in the current study, we evaluated whether prolongation of infusion time would be useful for ameliorating the nephrotoxicity of NDP at a therapeutic dose level.

\section{Materials and Methods}

\section{Chemicals}

Nedaplatin [cis-diammineglycolatoplatinum (II), NDP] was obtained from our laboratory (Shionogi \& Co., Ltd., Osaka, Japan).

\section{Animals and housing conditions}

All experimental procedures were conducted after 
approval of the study by the Institutional Animal Care and Use Committee of Shionogi Research Laboratories. All experiments were performed on 8-week-old male Wistar rats (Japan SLC Inc., Shizuoka, Japan). The animals were housed in plastic cages in an environment-controlled room kept at $23 \pm 1{ }^{\circ} \mathrm{C}, 45-65 \%$ humidity, with a 12-hour lighting period (light on: 8:00-20:00). All animals were allowed free access to food (autoclaved rat chow CA-1, Clea Japan Inc., Tokyo, Japan) and water (sterilized city water via an automatic water supplying system).

\section{Experimental design}

The day of administration was designated as Day 0 . NDP was dissolved in 5\% xylitol solution before use. NDP was injected intravenously in a single dose of $12 \mathrm{mg} / \mathrm{kg}$ with the following infusion times: a bolus injection (Bolus-NDP group, $\mathrm{n}=6$ ), 1-hour or 4-hour continuous infusions (1hIFor 4 hIF-NDP groups, $\mathrm{n}=6$ or 5 , respectively). Control rats received the vehicle alone under the corresponding infusion conditions (Bolus-, 1hIF- or 4hIF-Cont groups, $\mathrm{n}=4$, respectively). For the bolus injection, NDP $(2 \mathrm{~mL} / \mathrm{kg}$, at a concentration of $6 \mathrm{mg} / \mathrm{mL}$ ) was injected rapidly via the tail vein. For the continuous infusion, the NDP solution $(6 \mathrm{mg}$ / $\mathrm{mL}$ ) was further diluted with the vehicle at a concentration of $0.6 \mathrm{mg} / \mathrm{mL}$, which was infused through an indwelling needle placed in the caudal vein at a constant flow rate of 20 or 5 $\mathrm{mL} / \mathrm{kg} / \mathrm{h}$ for 1 or 4 hours, respectively. Body weight, residual amounts of food (Days 0 to 3 ) or water supplied (Day 3 only) on the previous day were measured daily in the morning. After the collection of a urine sample on the day of the sacrifice (Day 3), each animal was euthanized by exsanguination after cutting both the abdominal aorta and vena cava under deep anesthesia with pentobarbital sodium, and the kidneys and femurs (including bone marrow) were removed.

\section{Urinalysis}

Each animal was maintained in individual metabolic cages on Day 3, and consecutive 4-hour urine samples were collected under fasting and no-watering conditions. After urine volume was measured and color was observed, specific gravity (digital refractometer UR-S, Atago, Tokyo, Japan) was measured. The $\mathrm{pH}$, protein, glucose, ketone bodies, urobilinogen, bilirubin, and occult blood were determined using Multistix and an automatic analyzer Clinitek 200+ (Bayer Medical Ltd., Tokyo, Japan).

\section{Bone marrow analysis}

The number of nucleated bone marrow cells (NBMC) in the right femur was determined according to the following methods modified from a previous report ${ }^{9}$. Briefly, after the femur was removed and freed of soft tissue attachments, the epiphyseal cap was detached from the proximal end and the extreme distal tip of each epicondyle was cut off. A suspension of the bone marrow was prepared by injecting $1.5 \%$ acetic acid solution into the femurs to lyse erythrocytes and flush out cells. Nucleated cells in the suspension were counted using an automatic blood cell analyzer (T540, Beckman Coulter, Inc., Fullerton, CA, USA), and the number of NBMC per the bone marrow weight of a femur $\left(\times 10^{6} / \mathrm{mg}\right)$ was then calculated.

\section{Histopathological examination}

For light microscopic examination, kidney samples were fixed in $10 \%$ neutral buffered formalin, dehydrated in alcohol and embedded in paraffin. The left femur samples were decalcified in a mixture of $10 \%$ formic acid and formalin, before the specimens were prepared. Paraffin sections were prepared and stained by a routine method with hematoxylin and eosin (H\&E). The histopathological changes were graded by a pathologist in a blind and random manner according to the following criteria: (a) renal tubular lesions: \pm , very slight, $1-10 \%$ tubules affected within the kidney section; +, slight, 10-25\%; 2+, moderate, 25-50\%; $3+$, severe, over $50 \%$ tubules; (b) bone marrow hypocellularity: \pm , very slight; + , slight; $2+$, moderate; $3+$, severe. All specimens were further reviewed by another pathologist for confirmation of the results.

\section{TUNEL assay for apoptosis}

A TUNEL assay was performed on kidney sections from the NDP-treated groups using an ApopTag ${ }^{\circledR}$ Peroxidase In Situ Apoptosis Detection Kit (Chemicon International, Inc., Temecula, CA, USA) according to manufacturer's protocols. Briefly, the kidney sections were deparaffinized and rehydrated, followed by incubation with proteinase K (Dako Corporation, Carpinteria, CA, USA) for $15 \mathrm{~min}$ at room temperature, and immersion in PBS ( $\mathrm{pH} 7.4$ ) containing $3 \% \mathrm{H}_{2} \mathrm{O}_{2}$ to inactivate endogenous peroxidase. Equilibration buffer was applied to the sections for $30 \mathrm{~min}$ at room temperature, followed by reaction with TdT enzyme and reaction buffer at $37^{\circ} \mathrm{C}$ for $60 \mathrm{~min}$. Avidin-horseradish peroxidase solution was applied to the sections, and the staining was visualized with diaminobenzidine. Sections were then counter-stained with Mayer's hematoxylin, dehydrated, and mounted. A semiquantitative analysis was performed by counting the number of TUNEL-positive cells per field at $\times 400$ magnification in both the renal cortex and papilla. The mean numbers of stained cells in 20 fields in each renal zone of the kidney section were expressed as the number of TUNEL-positive cells.

\section{Statistical analysis}

All numerical data were expressed as mean \pm standard error (S.E.). Data on body weight, food consumption, water consumption, urinalysis (urine volume, specific gravity, $\mathrm{pH}$ ), NBMC, and the TUNEL assay were analyzed by Tukey's multiple comparison test. Results were considered significant when the $p$ value was less than 0.05 .

\section{Results}

Mortality, body weight and food consumption

One of the 6 animals in the Bolus-NDP group died on 
A

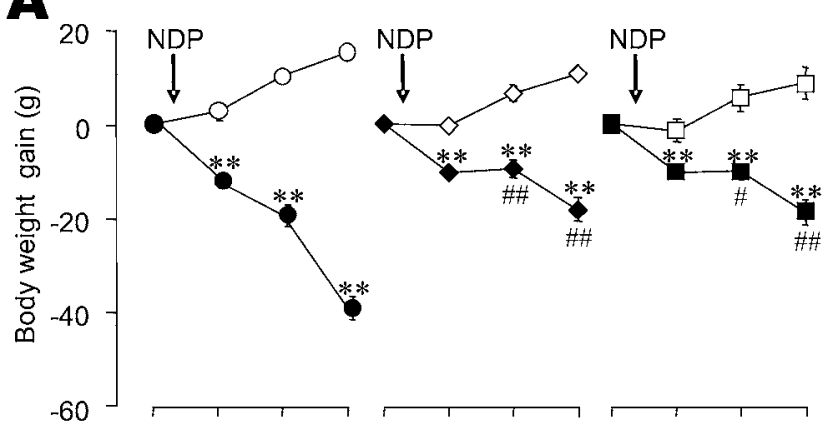

B

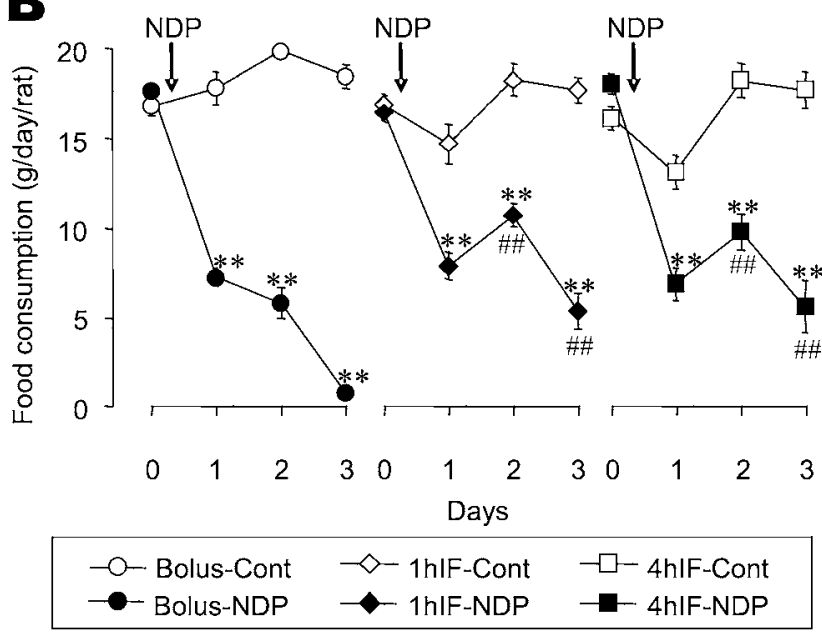

Fig. 1. Body weight gain and food consumption in rats treated with a single intravenous dose of $12 \mathrm{mg} / \mathrm{kg}$ nedaplatin (NDP) as a bolus, 1-hour (1hIF), or 4-hour infusion (4hIF). Shown are body weight (A) and daily food consumption (B). **: Significant difference from corresponding control groups by Tukey's multiple comparison test $(p<0.01)$. \#, \#\#: Significant difference from bolus nedaplatin-treated group by Tukey's multiple comparison test $(p<0.05$ and 0.01). Data are expressed as mean \pm S.E. ( $(n=4$ to 6$)$.

day 3. No deaths were observed in the other groups. In all the NDP-treated groups, there was a significant decrease in body weight (Fig. 1A) associated with a decrease in food consumption (Fig. 1B) throughout the study period, which was partially reduced by continuous infusion.

\section{Urinary parameters}

In all the NDP-treated groups, there was a tendency for urine volume to decrease with or without statistical significance compared with the corresponding control group (Fig. 2B) which was associated with a decrease in water consumption (Fig. 2A). In the Bolus- and 1hIF-NDP groups, urine specific gravity showed a tendency to decrease without statistical significance compared with the corresponding control group, while no abnormality was observed in the 4hIF-NDP group (Fig. 2C). In the Bolus-NDP group, low urine $\mathrm{pH}$ (5.0 to 5.5), glucosuria (2+ to $4+, 250$ to $1,000 \mathrm{mg}$ / $\mathrm{dL})$ and occult blood $(1+$ to $2+)$ were detected, while


Fig. 2. Water consumption, urinary volume and specific gravity in rats treated with a single intravenous dose of $12 \mathrm{mg} / \mathrm{kg}$ nedaplatin (NDP) as a bolus, 1-hour (1hIF), or 4-hour infusion (4hIF). Shown are water consumption (A), urinary volume (B) and osmolality (C). **: Significant difference between each group by Tukey's multiple comparison test $(p<0.01)$. Data are expressed as mean \pm S.E. $(n=4$ to 6 ).

abnormalities in these parameters were observed in neither the $1 \mathrm{hIF}$ - nor the $4 \mathrm{hIF}-\mathrm{NDP}$ groups (normal range of data: 6.0 to 7.0 for $\mathrm{pH}$, negative for glucose and occult blood). No abnormalities attributable to NDP-treatment were observed in the other urinary parameters.

\section{Bone marrow analysis}

There was no significant difference between the $1 \mathrm{hIF}$ and 4hIF-NDP groups in the number of NBMC, while the numbers in both the $1 \mathrm{hIF}$ - and $4 \mathrm{hIF}-\mathrm{NDP}$ groups were significantly higher than that in the Bolus-NDP group (Table 1). 
Table 1. Number of Nucleated Bone Marrow Cells in Rats Treated with a Single Intravenous Dose of $12 \mathrm{mg} / \mathrm{kg}$ Nedaplatin as a Bolus, 1-hour, or 4-hour Infusion

\begin{tabular}{llll}
\hline \multirow{2}{*}{ Groups } & \multicolumn{3}{c}{ Dosing protocols } \\
\cline { 2 - 4 } & \multicolumn{1}{c}{ Bolus } & 1-hour infusion & 4-hour infusion \\
\hline Control $(0 \mathrm{mg} / \mathrm{kg})$ & $2.13 \pm 0.05$ & $2.19 \pm 0.04$ & $2.20 \pm 0.05$ \\
Nedaplatin $(12 \mathrm{mg} / \mathrm{kg})$ & $0.98 \pm 0.09^{* *}$ & $1.44 \pm 0.06^{* *}, \# \#$ & $1.38 \pm 0.11^{* *}$, \#\# \\
\hline
\end{tabular}

Rats were sacrificed 3 days after drug administration. All data are expressed as mean \pm S.E. ( $n=4$ to 6$)$.

**: Significant difference from corresponding control groups, $p<0.01$.

\#\#: Significant difference from bolus nedaplatin-treated group, $p<0.01$.

Table 2. Histopathology of Rat Kidneys and Femurs 3 Days after Treatment with a Single Intravenous Dose (Bolus, 1-hour Infusion, or 4-hour Infusion) of $12 \mathrm{mg} / \mathrm{kg}$ Nedaplatin

\begin{tabular}{lccc}
\hline & \multicolumn{2}{c}{ Nedaplatin $12 \mathrm{mg} / \mathrm{kg}$} \\
\hline Histopathological findings & Bolus & 1-hour infusion & 4-hour infusion \\
\hline No. of animals examined & $5(1)^{\mathrm{a})}$ & 6 & 5 \\
Renal cortex (including corticomedullary zone) & & 0 & 0 \\
$\quad$ Focal necrosis of tubular epithelium & $4(2+), 1(+)$ & $6( \pm)$ & $3( \pm)$ \\
$\quad$ Single cell necrosis of tubular epithelium & $4(2+), 1(+)$ & 0 & 0 \\
$\quad$ Tubular degeneration & $4(2+), 1(+)$ & $6( \pm)$ & 0 \\
Renal papilla & $4(+), 1( \pm)$ & $1(+), 5( \pm)$ & 0 \\
$\quad$ Single cell necrosis of collecting duct epithelium and epithelium covering papilla & $3( \pm)$ & $1(2+), 5(+)$ & $1(2+), 4(+)$ \\
$\quad$ Hyaline droplet in collecting duct epithelium and epithelium covering papilla & $4(2+), 1(+)$ & 1
\end{tabular}

Rats were sacrificed 3 days after drug administration. Figures represent the number of rats affected. The histopathological changes were arbitrarily scored as follows: \pm , very slight; + , slight; $2+$, moderate; $3+$, severe. All control groups were excluded from this table as no abnormal pathological changes were noted. a) One of the 6 rats died during the study period and was not examined histopathologically due to advanced autolysis.

\section{Histopathological examination}

As shown in Table 2, all rats in the Bolus-NDP group displayed slight to moderate renal tubular lesions, such as focal necrosis and degenerative appearances manifesting loss of brush borders and intracytoplasmic abnormally large vacuoles, and single cell necrosis, characterized by shrunken cytoplasm with pyknotic and fragmented nuclei in the tubular epithelium of the renal cortex and corticomedullary zone (Fig. 3A). In addition, morphological abnormalities caused by NDP were not restricted to the renal cortex, and single cell necrosis and hyaline droplets were observed in the collecting duct epithelium and the epithelium covering the renal papilla (Fig. 3B).

In comparison with the Bolus-NDP group, there was a clear reduction in the incidence and the severity of renal lesions in the 1hIF- and 4hIF-NDP groups (Fig. 3C and D). In particular, focal necrosis of the cortical renal tubules was not observed in both the $1 \mathrm{hIF}$ - and 4hIF-NDP groups. The incidence and the severity of renal lesions in the $1 \mathrm{hIF}$ - and 4hIF-NDP groups, such as single cell necrosis and tubular degeneration, were substantially reduced in comparison with the Bolus-NDP group. In the 4hIF-NDP group, only a few animals showed single cell necrosis graded as very slight in the renal cortex.

In contrast, all of the NDP-treated rats showed slight to moderate hypocellularity in the bone marrow. In comparison with the Bolus-NDP group, the incidence of the bone marrow lesion showed a tendency to reduce in 1hIFand 4hIF-NDP groups. However, there was no clear reduction in the severity of the lesions in the 4hIF-NDP group compared with that in the 1hIF-NDP group.

\section{TUNEL assay for apoptosis}

As shown in Fig. 4, the numbers of the TUNELpositive cells in the $1 \mathrm{hIF}$ - and 4hIF-NDP groups were significantly lower than that in the Bolus-NDP group in both the renal cortex and papilla. In the 4hIF-NDP group, the number of TUNEL-positive cells showed a tendency to decrease with or without statistical significance compared with that in the 1hIF-NDP group in both the renal cortex and papilla.

\section{Discussion}

Several platinum complexes possessing antitumor properties have been synthesized. Cisplatin [cisdiamminedichloroplatinum (II), CDDP] is one of the most potent agents used in chemotherapy for various types of cancer. However, despite its effectiveness as an antitumor drug, its clinical use is restricted by its severe 

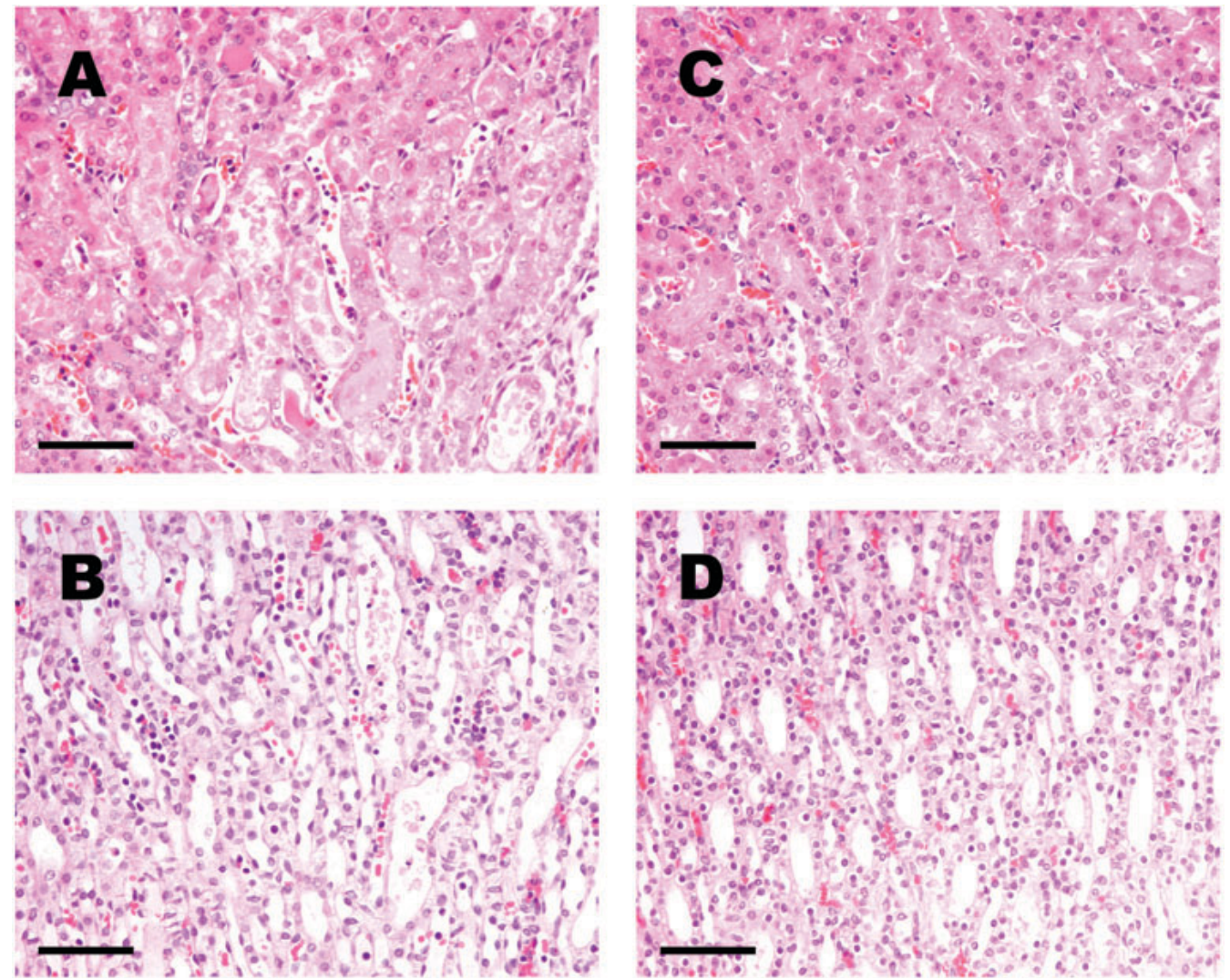

Fig. 3. Histopathological changes in rats 3 days after treatment with a single intravenous dose of $12 \mathrm{mg} / \mathrm{kg}$ nedaplatin (NDP) as a bolus or 4hour infusion (4hIF). Shown are the renal cortex (A) and papilla (B) of a Bolus-NDP rat, and the renal cortex (C) and papilla (D) of a 4hIF-NDP rat. Note that the severity of the renal tubular lesions was clearly reduced by the prolongation of infusion time both in the renal cortex and papilla. H\&E, bars indicate $70 \mu \mathrm{m}$.

nephrotoxicity, which can lead to acute renal failure ${ }^{10-12}$. To resolve the problem of this dose-limiting nephrotoxicity, several investigators have attempted to alter the schedule of administration or find ways to ameliorate it in experimental animals with the ultimate aim of application to humans. These efforts have led to optimal dosing protocols designed to minimize the nephrotoxicity, including longer continuous infusions ${ }^{13,14}$ and the use of hydration and osmotic diuresis $^{15-17}$. Nevertheless, approximately one-third of patients receiving CDDP still develop evidence of acute renal failure ${ }^{16,18,19}$.

A wealth of toxicological data is available concerning CDDP-induced nephrotoxicity. Relevant perhaps to the nephrotoxicity of CDDP are the observations that the kidney retains platinum to a greater extent than other organs and is the predominant excretory organ for $\mathrm{CDDP}^{22-24}$. Several experimental studies have confirmed that CDDP primarily affects the lower straight part (S3 segment) of the proximal tubules, the principal site of platinum accumulation, in the corticomedullary zone in rats ${ }^{25,26}$. This preferential accumulation of platinum seems to be a major cause of the nephrotoxicity of CDDP.

NDP is a promising antineoplastic platinum complex with reduced nephrotoxicity. Kawai et al. ${ }^{27}$ showed that renal accumulation of platinum when cisplatin was

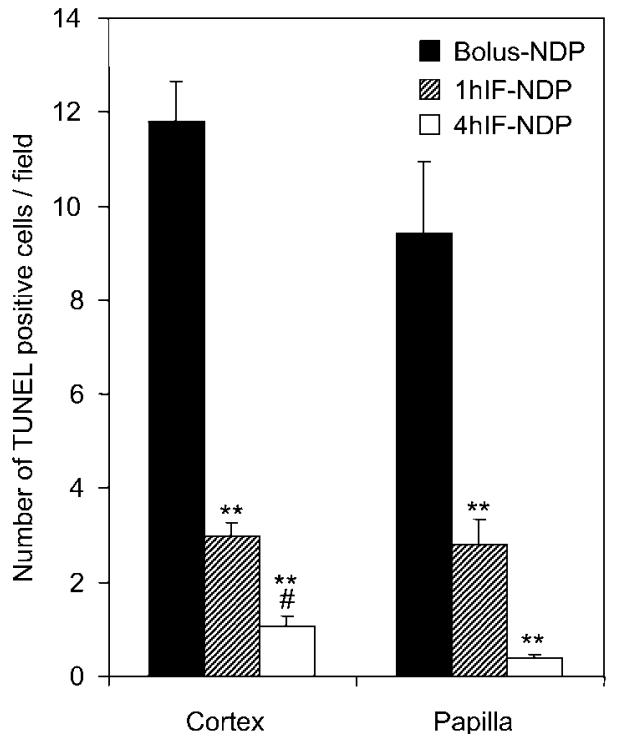

Fig. 4. Number of TUNEL-positive cells in the kidneys of rats treated with a single intravenous dose of $12 \mathrm{mg} / \mathrm{kg}$ nedaplatin (NDP) as a bolus, 1-hour (1hIF), or 4-hour infusion (4hIF). The mean number of stained cells in 20 fields in each kidney was expressed as the number of TUNEL-positive cells in each renal cortex and papilla. **: Significant difference from Bolus-NDP group, $p<0.01$. \#: Significant difference from 1hIF-NDP group, $p<0.05$. 
administered at $3.75 \mathrm{mg} / \mathrm{kg}$ was similar to that of NDP administered at $24 \mathrm{mg} / \mathrm{kg}$, and that both drugs at these two doses induced similar effects in the kidney. It was inferred, therefore, that the nephrotoxicity of NDP was lower than that of CDDP due to lower accumulation of platinum. Sugeno et $a l .{ }^{28}$ reported that the plasma levels of platinum decreased in a bi-exponential manner in male rats after a single intravenous bolus dose of $3 \mathrm{mg} / \mathrm{kg} \mathrm{NDP}$; the half-lives of the $\alpha$ - and $\beta$-phase were 1.7 and 14.5 minutes and most of the plasma platinum was of the free form. They also demonstrated that approximately over $85 \%$ of the total dose of platinum was excreted in urine within 6 hours after a single intravenous dose. Therefore, NDP is rapidly excreted from blood into urine after dosing.

In the current study, a single bolus intravenous dose of $12 \mathrm{mg} / \mathrm{kg}$ NDP was administered, which corresponds to an approximate $62 \mathrm{mg} / \mathrm{m}^{2}$ body surface area based on a method previously published ${ }^{20}$. However, there were several morphological abnormalities in the kidney treated with NDP as a single bolus dose. This result suggests that NDP also has the potential to cause nephrotoxicity at a dose within the therapeutic range for clinical use $\left(50-100 \mathrm{mg} / \mathrm{m}^{2}\right)^{21}$.

In the present study, we also evaluated whether continuous infusion could ameliorate NDP-induced nephrotoxicity. We found that morphological abnormalities in kidneys treated with NDP by 4-hour infusion were milder than those given NDP by bolus or 1-hour infusion, in spite of the same dose being given. This finding indicates that the nephrotoxicity of NDP can be reduced by prolongation of its infusion time. On the other hand, prolongation of the infusion time of NDP dosing had no clear effects on the myelotoxicity. Although pharmaco/toxicokinetic data were not available under the current experimental conditions, we consider that nephrotoxicity attributable to NDP was probably avoided by prevention of temporary elevation of urine platinum concentration because of the prolonged infusion time.

In summary, our present results provide new evidence that prolongation of infusion time is effective at minimizing the nephrotoxicity of NDP. Our findings offer valuable information for the consideration of the clinical therapeutic use of NDP.

\section{References}

1. Fukuda M, Shinkai T, Eguchi K, Sasaki Y, Tamura T, Ohe Y, Kojima A, Oshita F, Hara K, and Saijo N. Phase II study of (glycolato-O,O) diammineplatinum (II), a novel platinum complex, in the treatment of non-small-cell lung cancer. Cancer Chemother Pharmacol. 26: 393-396. 1990.

2. Ota K, Wakui A, Majima H, Niitani H, Inuyama Y, Ogawa M, Ariyoshi Y, Yoshida O, Taguchi T, and Kimura I. Phase I study of a new platinum complex 254-S, cis-diammine (glycolato)-platinum (II). Gan To Kagaku Ryoho. 19: 855861.1992 (In Japanese).

3. Taguchi T, Wakui A, Nabeya K, Kurihara M, Isono K, Kakegawa T, and Ota K. A phase II clinical study of cisdiammine glycolato platinum, 254-S, for gastrointestinal cancers. Gan To Kagaku Ryoho. 19: 483-488. 1992 (In Japanese).

4. Inuyama Y, Miyake H, Horiuchi M, Hayasaki K, Komiyama $\mathrm{S}$, and Ota K. A late phase II clinical study of cis-diammine glycolato platinum, 254-S, for head and neck cancers. Gan To Kagaku Ryoho. 19: 871-877. 1992.

5. Akaza H, Togashi M, Nishio $Y$, Miki $T$, Kotake $T$, Matsumura Y, Yoshida O, and Aso Y. Phase II study of cisdiammine(glycolato)platinum, 254-S, in patients with advanced germ-cell testicular cancer, prostatic cancer, and transitional-cell carcinoma of the urinary tract. Cancer Chemother Pharmacol. 31: 187-192. 1992.

6. Kato T, Nishimura H, Yakushiji M, Noda K, Terashima Y, Takeuchi S, Takamizawa H, Suzuki M, Arai M, and Ota M. Phase II study of 254-S (cis-diammine glycolato platinum) for gynecological cancer. Gan To Kagaku Ryoho. 19: 695701. 1992 (In Japanese).

7. Ota K. Nedaplatin. Gan To Kagaku Ryoho. 23: 379-387. 1996 (In Japanese).

8. Uehara $\mathrm{T}$, Watanabe $\mathrm{H}$, Itoh $\mathrm{F}$, Inoue $\mathrm{S}$, Koshida $\mathrm{H}$, Nakamura M, Yamate J, and Maruyama T. Nephrotoxicity of a novel antineoplastic platinum complex, nedaplatin: a comparative study with cisplatin in rats. Arch Toxicol. 79: 451-460. 2005.

9. Chervenick PA, Boggs DR, Marsh JC, Cartwright GE, and Wintrobe MM. Quantitative studies of blood and bone marrow neutrophils in normal mice. Am J Physiol. 215: 353-360. 1968.

10. Goldstein RS and Mayor GH. Minireview. The nephrotoxicity of cisplatin. Life Sci. 32: 685-690. 1983.

11. Arany I and Safirstein RL. Cisplatin nephrotoxicity. Semin Nephrol. 23: 460-464. 2003.

12. Hartmann JT and Lipp HP. Toxicity of platinum compounds. Expert Opin Pharmacother. 4: 889-901. 2003.

13. Posner MR, Skarin AT, Clark J, and Ervin TJ. Phase I study of continuous-infusion cisplatin. Cancer Treat Rep. 70: 847850. 1986.

14. Bozzino JM, Prasad V, and Koriech OM. Avoidance of renal toxicity by 24 -hour infusion of cisplatin. Cancer Treat Rep. 65: 351-352. 1981.

15. Cornelison TL and Reed E. Nephrotoxicity and hydration management for cisplatin, carboplatin, and ormaplatin. Gynecol Oncol. 50: 147-158. 1993.

16. Santoso JT, Lucci JA 3rd, Coleman RL, Schafer I, and Hannigan EV. Saline, mannitol, and furosemide hydration in acute cisplatin nephrotoxicity: a randomized trial. Cancer Chemother Pharmacol. 52: 13-18. 2003.

17. Ali $\mathrm{BH}$ and $\mathrm{Al}$ Moundhri MS. Agents ameliorating or augmenting the nephrotoxicity of cisplatin and other platinum compounds: a review of some recent research. Food Chem Toxicol. 44: 1173-1183. 2006.

18. Meyer KB and Madias NE. Cisplatin nephrotoxicity. Miner Electrolyte Metab. 20: 201-213. 1994.

19. Taguchi T, Nazneen A, Abid MR, and Razzaque MS. Cisplatin-associated nephrotoxicity and pathological events. Contrib Nephrol. 148: 107-121. 2005.

20. Freireich EJ, Gehan EA, Rall DP, Schmidt LH, and Skipper HE. Quantitative comparison of toxicity of anticancer agents in mouse, rat, hamster, dog, monkey, and man. Cancer Chemother. 50: 219-244. 1966.

21. Daugaard G and Abildgaard U. Cisplatin nephrotoxicity. A review. Cancer Chemother Pharmacol. 25: 1-9. 1989. 
22. Smith PHS and Taylor DM. Distribution and retention of the antitumour agent 195M-PT-cis-dichlorodiammineplatinum (II) in man. J Nucl Med. 15: 349-351. 1974.

23. Litterst CL, LeRoy AF, and Guarino AM. Disposition and distribution of platinum following parenteral administration of cis-dichlorodiammineplatinum(II) to animals. Cancer Treat Rep. 63: 1485-1492. 1979.

24. Fichtinger-Schepman AM, Vendrik CP, van DijkKnijnenburg WC, de Jong WH, van der Minnen AC, Claessen AM, van der Velde-Visser SD, de Groot G, Wubs KL, Steerenberg PA, Schornagel, JH, and Berends F. Platinum concentrations and DNA adduct levels in tumors and organs of cisplatin-treated LOU/M rats inoculated with cisplatin-sensitive or -resistant immunoglobulin M immunocytoma. Cancer Res. 49: 2862-2867. 1989.

25. Leibbrandt ME, Wolfgang GH, Metz AL, Ozobia AA, and
Haskins JR. Critical subcellular targets of cisplatin and related platinum analogs in rat renal proximal tubule cells. Kidney Int. 48: 761-770. 1995.

26. Townsend DM, Deng M, Zhang L, Lapus MG, and Hanigan MH. Metabolism of Cisplatin to a nephrotoxin in proximal tubule cells. J Am Soc Nephrol. 14: 1-10. 2003.

27. Kawai Y, Taniuchi S, Okahara S, Nakamura M, and Gemba M. Relationship between cisplatin or nedaplatin-induced nephrotoxicity and renal accumulation. Biol Pharm Bull. 28: 1385-1388. 2005.

28. Sugeno K, Mizojiri K, Okabe H, Esumi Y, Takaichi M, and Okada Y. Studies on the disposition of a new antineoplastic agent, cis-diammine(glycolato)platinum (254-S) (I) Distribution and excretion of platinum in rats. Iyakuhin Kenkyu. 22: 231-242. 1991 (In Japanese). 